\title{
DESPRENDIMIENTO DEL VÍTREO POSTERIOR Y VITREOLISIS FARMACOLÓGICA: LA NUEVA ERA DE LA VITRECTOMÍA ENZIMÁTICA
}

\section{POSTERIOR VITREOUS DETACHMENT AND PHARMACOLOGIC VITREOLYSIS: THE NEW AGE OF ENZYMATIC VITRECTOMY}

\author{
DÍAZ-LLOPIS $\mathrm{M}^{1}$, CERVERA E ${ }^{1}$
}

El desprendimiento del vítreo posterior (DVP) es un proceso degenerativo por el que la hialoides posterior y el córtex vítreo, asociado a un gel vítreo generalmente en proceso de licuefacción progresiva, se despega de la retina. Es un proceso senil, podríamos considerar casi fisiológico con la edad y mucho más frecuente en miopes. Estudios postmorten han demostrado que a los 80-90 años más del $50 \%$ del gel vítreo esta licuado. Otros de los factores implicados parecen ser la ingesta elevada de vitamina B6 y también la menopausia, pues la síntesis y metabolismo de los proteinglicanos del vítreo está modulado por las hormonas sexuales, actuando los niveles altos de estrógenos como factor protector del DVP (1).

Mucho más conocida es la ultraestructura que el metabolismo del vítreo. Los estudios de Sebag (2) ya demostraron que el gel vítreo es en un $98 \%$ agua. Sus macromoléculas importantes son el colágeno y el ácido hialurónico, lo que le confiere su estructura tridimensional, junto a proteoglicanos, glicoproteinas y otras moléculas. El ácido hialurónico tiene gran capacidad de hidratación y es el responsable principal de la viscosidad del gel. Tanto el ácido hialurónico como el colágeno - fundamentalmente el colágeno tipo II- se concentran especialmente en la zona periférica del vítreo o córtex. Por ello el cortex vítreo es más denso y consistente que la porción central. La interfase vitreoretiniana, zona virtual de adhesión entre la membrana limitante interna retiniana y la hialoides posterior que delimita el córtex vítreo, está mantenida principalmente por las moléculas fibronectina, laminina y colágena tipo IV, que actúan como un verdadero pegamento bio- lógico. Fine y Spaide (3) demostrando la existencia de un bolsillo vítreo precortical y los canales de comunicación interna por los que se deslizan hacia la mácula los fármacos tras su inyección intravítrea (triamcinolona, antibióticos anti-VEGF, antibióticos, etc.) han confirmado los clásicos esquemas teóricos de la bursa premacular.

El metabolismo del vítreo permanece en gran parte desconocido. El papel de los hialocitos del vítreo no está del todo claro: con su características funcionales como macrófagos, actividad fibrinolítica y de la contracción del colágeno vítreo, están directamente implicados en el mantenimiento del vítreo claro y avascular, y muy posiblemente también en la aparición del DVP. Algunas moléculas procolágenas que son producidas a nivel vítreo de manera natural, siguen segregándose a pesar de la vitrectomía, lo que explicaría en parte la reproliferación de membranas o la persistencia de edemas maculares o de cierres incompletos de los agujeros maculares a pesar de realizar vitrectomía incluyendo el pelado de la membrana limitante interna interna (4).

Pero ¿para qué nos sirve tener vítreo? Es universalmente conocido el perverso efecto a que conduce que el vítreo permanezca fuertemente adherido a la retina y organizado, y lo deseable que se hubiera desprendido antes de una manera lenta, progresiva y controlada: 1) Recidivas postdesprendimiento de retina con o sin proliferación vitreoretiniana. 2) Desprendimientos de retina en patologías con vítreos organizados —síndrome de Stickler, etc. - 3) Bilateralización del desgarros gigantes 4) Persistencia y cronificación de edemas maculares (diabético, oclusión venosa, uveítis, pseudofáquico síndrome de

\footnotetext{
1 Doctor en Medicina. Hospital General Universitario de Valencia. Departamento de Oftalmología. Unidad Docente de Oftalmología. Facultad de Medicina. Universidad de Valencia.

E-mail: Manuel.Diaz@uv.es
} 
Irvine-Gass). 5) Síndrome de tracción macular miópica. 6) Membrana epirretinianas maculares 6) Aparición de agujeros maculares y su progresión de incompletos a completos. 7) Progresión de la retinopatía diabética proliferante por tracción vítrea de los neovasos. Todo ello nos habla del «bondadoso» y efecto profiláctico de «padecer» el DVP lo antes posible.

Pero el DVP por un lado tan beneficioso es el origen fisiopatológico de patologías graves -el desprendimiento de retina regmatógeno-- o del empeoramiento de las ya existentes, como por ejemplo la transformación de un agujero macular incompleto en completo, o de un edema macular cistoideo en una agujero macular lamelar.

El DVP bruscamente sintomático, patología que popularmente se asocia al «aumento brusco de moscas volantes», debemos recordar que en casi un 2$5 \%$ de los casos asocia un opérculo o desgarro retiniano si tenemos la paciencia de dilatar al paciente, y explorarle detalladamente los $360^{\circ}$ de la periferia retiniana bajo biomicroscopía. Por otro lado, todos los pacientes con DVP unilateral deben ser advertidos sobre el riesgo de su aparición en el ojo contralateral en un plazo corto: desde $25 \%$ durante el primer año hasta más del $90 \%$ antes de los 3 años, no estando demostrado ni siendo universalmente aceptado en la literatura el tratamiento profiláctico de lesiones «predisponentes asintomáticas» de la periferia retiniana $(4,5)$.

En definitiva, la idea de que podría ser beneficioso una actuación profiláctica y terapéutica poco invasiva y con bajo riesgo «induciendo de manera controlada la aparición de un DVP artificialmente» ha ido adquiriendo poco a poco posibilidades clínicas, y así es como nacen la genialidad de utilizar el anillo de succión del LASIK para inducirlo (6) o el concepto de la vitreolisis farmacológica del vítreo humano. La vitreolisis farmacológica por tanto es considerada como una modalidad terapéutica que pretende alterar químicamente la estructura del vítreo y debilitar la adhesión vitreorretiniana para desprender de forma segura el vítreo posterior de la retina interna (7).

Han sido ensayados diversos fármacos proteolíticos contra los componentes fundamentales de la adhesión vitreorretinal y con capacidad enzimática directa o indirectamente degradatoria de sustancias implicadas en el mantenimiento de la estructura y matriz extracelular colágena vítrea que actúan acelerando su licuefacción, como son la dispasa, hialuronidasa, condroitinasa, activador tisular del plasminógeno, uroquinasa, estreptoquinasa, natokinasa, colagenasa, plasmina y microplasmina (8).

La natokinasa (Subtisilin NAT ${ }^{\circledR}$ ), hialuronidasa $\left(\right.$ Vitrase $^{\circledR}$ ) y la microplasmina (Trombogenic ${ }^{\circledR}$ ) estan en fases II y III de ensayo clínico, y previsiblemente posterior comercialización. De todas ellas con mucho la mejor estudiada es la plasmina. La plasmina es una proteasa aislable del propio suero del paciente, y ya ha sido usada como coadyuvante 15 minutos previamente a la vitrectomía quirúrgica facilitando la cirugía de agujeros maculares, y edemas maculares diabéticos. Uno de los principales problemas actuales para su uso es su inestabilidad en el tiempo, amén de su costosa, laboriosa y sofisticada técnica de preparación.

Si este método llega a desarrollarse clínicamente, es decir conseguir despegar y licuar el vítreo con una simple inyección intravítrea, podemos estar en un futuro no muy lejano ante un importante cambio de paradigma de nuestra actual concepción de la cirugía vítrea que hasta el día de hoy hemos conocido: el nacimiento de la vitrectomía enzimática.

\section{BIBLIOGRAFÍA}

1. Chuo JY, Lee TY, Hollands H, Morris AH, Reyes RC, Rossiter JD, et al. Risk factors for posterior vitreous detachment: a case-control study. Am J Ophthalmol 2006; 142: 931-937.

2. Sebag J. The vitreous-structure, function, and pathobiology. New York: Springer-Verlag; 1989.

3. Fine $H F$, Spaide RF. Visualization of the posterior precortical vitreous pocket in vivo with triamcinolone. Arch Ophthalmol 2006; 124: 1663.

4. Hikichi T. Time course of posterior vitreous detachment in the second eye. Curr Opin Ophthalmol 2007; 18: 224-227.

5. Lewis H. Peripheral retinal degenerations and the risk of retinal detachment. Am J Ophthalmol 2003; 136: 155160.

6. Venkatesh P, Vajpayee RB, Garg S. Induction of posterior vitreous separation using LASIK suction ring may have a potential role in the management of diabetic macular edema. Med Hypotheses 2006; 66: 1137-1139.

7. Gandorfer A. Pharmacologic vitreolysis. Dev Ophthalmol 2007; 39: 149-156.

8. Trese MT. Enzymatic vitreous surgery. Semin Ophthalmol 2000; 15: 116-121. 